\title{
Exploration of the close chemical space of tryptophan and tyrosine reveals importance of hydrophobicity in CW-photo-CIDNP performances
}

\author{
Felix Torres, Alois Renn, and Roland Riek \\ Laboratory of Physical Chemistry, ETH Zürich, Zurich, 8093, Switzerland \\ Correspondence: Roland Riek (roland.riek@phys.chem.ethz.ch) \\ Received: 5 January 2021 - Discussion started: 20 January 2021 \\ Revised: 23 April 2021 - Accepted: Accepted on 26 - Published: 12 May 2021
}

\begin{abstract}
Sensitivity being one of the main hurdles of nuclear magnetic resonance (NMR) can be gained by polarization techniques including chemically induced dynamic nuclear polarization (CIDNP). Kaptein demonstrated that the basic mechanism of the CIDNP arises from spin sorting based on coherent electron-electron nuclear spin dynamics during the formation and the recombination of a radical pair in a magnetic field. In photoCIDNP of interest here the radical pair is between a dye and the molecule to be polarized. Here, we explore continuous-wave (CW) photo-CIDNP (denoted CW-photo-CIDNP) with a set of 10 tryptophan and tyrosine analogues, many of them newly identified to be photo-CIDNP active, and we observe not only signal enhancement of 2 orders of magnitude for ${ }^{1} \mathrm{H}$ at $600 \mathrm{MHz}$ (corresponding to 10000 times in measurement time) but also reveal that polarization enhancement correlates with the hydrophobicity of the molecules. Furthermore, the small chemical library established indicates the existence of many photo-CIDNP-active molecules.
\end{abstract}

\section{Introduction}

Despite decades of development and impressive technological improvements, sensitivity remains the main hurdle of nuclear magnetic resonance (NMR) spectroscopy and imaging (Ardenkjaer-Larsen et al., 2015). Chemically induced dynamic nuclear polarization (CIDNP) enhances the sensitivity of NMR thanks to out-of-Boltzmann nuclear spin polarization. The first anomalous lines related to CIDNP were serendipitously observed in 1967 independently by Bargon et al. (1967) and Ward and Lawler (1967). The radical pair mechanism was proposed by Kaptein and Oosterhoff (1969) and by Closs (1969) 2 years after and remains the cornerstone of the CIDNP theory ever since. Kaptein demonstrated that the polarization arises from the formation and the recombination of a radical pair in a magnetic field. The radicals can be generated in different ways such as heating, flash photolysis and photochemical reaction. The last generation mechanism is so-called photo-CIDNP and is the one presented in this work. In photo-CIDNP, light is shined into the sample where a photosensitizer is excited and can undergo intersystem crossing towards a triplet state. The triplet-state dye reacts with a molecule of interest $(M)$ and forms a radical pair after abstraction of an electron from that molecule. The newly formed radical pair is in a triplet state and cannot recombine due to the Pauli principle. The interplay of nuclear-spin-dependent electron intersystem crossing into a singlet state, allowing the electron back-transfer, yields different radical pair recombination kinetics depending on the nuclear spin state. Therefore, CIDNP can be used to study transient radicals that are too short lived for EPR (Closs and Trifunac, 1969; Morozova et al., 2008, 2007, 2005) to study protein structure (Kaptein et al., 1978) and folding (Hore et al., 1997; Mok et al., 2003; Mok and Hore, 2004), or to study the electron-transfer mechanism (Morozova et al., $2018,2008,2005,2003)$. The radical pair mechanism is extensively described in different papers that we recommend to the reader for a deeper understanding (Goez, 1995; Morozova and Ivanov, 2019; Okuno and Cavagnero, 2017; Kuhn, 2013). Robert Kaptein's key role in the development of the theory underlying the CIDNP mechanism is crystallized in the Kaptein rules which capture the theory of CIDNP into 
a simple equation in order to qualitatively analyze the sign of an anomalous CIDNP line (Kaptein, 1971). According to Kaptein's rules, considering a radical pair composed of molecules $a$ and $b$ the sign of the polarization on a nucleus $i$ belonging to a is predicted by the following equation:

$\Gamma_{\mathrm{ne}}=\mu \varepsilon \Delta g A_{i}$,

where $\Gamma_{\text {ne }}$ is the net polarization sign of the radical $a$, and $\mu$ and $\varepsilon$ are Boolean values. $\mu$ is positive when the radical is formed from a triplet precursor and negative otherwise. $\varepsilon$ is positive for recombination products and negative if the radical escaped or for the transfer reaction products. $\Delta g$ is the sign of the $g$ factor's difference between the two radicals, i.e., $g_{a}-g_{b}$, and $A_{i}$ is the hyperfine coupling constant sign of the considered nucleus $i$ in the radical $a$ which makes the reaction nuclear spin selective. Kaptein's rules equation predicts the sign of polarization and reflects the complex nature of the reaction path that yields out-of-Boltzmann spin polarization. Therefore, it can be used for a qualitative analysis of the photo-CIDNP products.

Extensive studies of the photo-CIDNP effect monitored by ultraviolet-absorbing dyes such as FMN (flavin mononucleotide) (Tsentalovich et al., 2002), bipyridyl (Tsentalovich et al., 2000) or 3,3',4,4'-tetracarboxybenzophenone (TCBP) (Morozova et al., 2011) using state-of-the-art time-resolved (TR) photo-CIDNP (Hore et al., 1981) elucidated a great understanding of the photo-CIDNP theory. However it has also been applied to a small list of target molecules (a dozen or so) such as tryptophan (TRP) and tyrosine (TYR) (Table S1). Therefore, the determinants of polarization of the individual dye-molecule pair systems including magnetic field dependency, $g$ factors, hyperfine couplings and timing are described for a very reduced number of systems. However, less extensively described molecules are also reported in the literature and draw the start of the endeavor to explore the chemical space of photo-CIDNP performances (Table S1). However, this number of molecules remains small and would benefit from being significantly increased.

In contrast to the physical-based approach, it is the focus of our attempt to elaborate on the chemical space of continuous-wave $(\mathrm{CW})$ photo-CIDNP-active molecules in order to bring $\mathrm{CW}$-photo-CIDNP towards a versatile and straightforward applicable tool in biomedical and biochemical research. Initially, our recent efforts have been to push the dye absorption towards more biocompatible wavelengths such as the near-infrared region $(650-900 \mathrm{~nm})$. In parallel, the performance of photo-CIDNP with a readily handled light source is a contemporary goal (Bernarding et al., 2018). These efforts yielded the discovery of the Atto Thio 12 (AT12) dye which monitored CW-photo-CIDNP experiments with a promising signal-to-noise enhancement (SNE) after laser irradiation at $450 \mathrm{~nm}$ (Sobol et al., 2019). Furthermore, the light source was an affordable continuous-wave laser which could be set up within a few minutes on different Bruker spectrometers: in our case $200 \mathrm{MHz}$ Avance,
$600 \mathrm{MHz}$ Avance III and $700 \mathrm{MHz}$ Avance Neo. On this journey to establish a CW-photo-CIDNP in biomedicine, a strong dependency of the photo-CIDNP SNE on the dye-molecule couple was observed. For instance, tryptophan is poorly polarized in the presence of AT12 but highly polarized in the presence of fluorescein, and tyrosine is highly polarized in the presence of AT12 and less well polarized in the presence of fluorescein (Okuno and Cavagnero, 2016; Sobol et al., 2019). These changing performances were attributed in an initial approximation to the chemical structures of the aromatic rings and the atoms in their close vicinity yielding different magnetic parameters, $g$ values and hyperfine coupling (HFC). This assumption was corroborated by the observation of anomalous line sign alternation for an oxidocyclization product of tryptophan while the dye monitoring the reaction was changed from AT12 to fluorescein (Torres et al., 2021). This observation is related to the $g$-factor difference between the two molecules as we shall see, and it is thus an elegant illustration of Kaptein's rules.

Moreover, in this work we show the importance of side chains in the intensity of the anomalous lines in continuouswave $(\mathrm{CW})$ photo-CIDNP experiments. Although the effect of the chemical modifications on the aromatic part of the molecules are well described by theory and confirmed experimentally (Kuprov et al., 2007; Kuprov and Hore, 2004), the effect of the nonaromatic moieties are considered to be conditioning the triplet-state dye quenching kinetics, as observed in time-resolved (TR) photo-CIDNP (Saprygina et al., 2014). While the exact mechanism of polarization can be only evaluated by TR-photo-CIDNP, in the context of biomedical NMR application aiming for the highest polarization at low micromolar molecule concentration, CW-photo-CIDNP appears to be the method of choice, suggesting the exploration of a CW-photo-CIDNP-based empirical approach indicated. This work reports on this approach by screening $10 \mathrm{com}-$ pounds with two different dyes.

\section{Results}

\subsection{On the Kaptein rule of the oxidocyclization product of tryptophan $\alpha$-hydroxypyrroloindole}

The distinct photo-CIDNP performances of the different dye-molecule couples was previously discussed in the literature (Sobol et al., 2019; Okuno and Cavagnero, 2016). For interest here, the tryptophan presented a higher signalto-noise enhancement (SNE) when polarized upon fluorescein (for chemical structure see Fig. 1) irradiation when compared with the dye AT12 (for chemical structure see Fig. 1) as shown in Fig. 1 and listed in Table 1, whereas tyrosine was better polarized in the presence of AT12 (Fig. 1) (Sobol et al., 2019). The differential effect of the dyes to trigger the radical pair mechanism has been further studied and yielded the serendipitous observation of $3 \alpha$-hydroxypyrroloindole (HOPI, for chemical structure see Fig. 1), which is an oxi- 
docyclization product of tryptophan that is highly polarized after irradiation in the presence of AT12 (Fig. 1, Table 1). The study of HOPI revealed surprising features such as different polarization yields between the cis and trans diastereoisomers and the sign alternation of the anomalous intensities depending on the dye used to form the radical pair (Torres et al., 2021). This sign alternation is assessed here in the light of the Kaptein rules (Eq. 1). In the case of the photoCIDNP reaction that is performed for all the experiments of this work, $\mu$ is positive since the radical pair is formed in a triplet state. Moreover, the polarized species are the recombination products of the radical pairs; thus, the parameter $\varepsilon$ is positive. Hence, the variable parameters are the hyperfine coupling when the molecule changes, e.g., from tryptophan to HOPI, and/or $\Delta g$. The $\Delta g$ can also alter when the dye used is changed. Hence, for the same molecule a sign change of the NMR signal upon switch from one dye to another is necessarily caused by an alternation of the sign of the $\Delta g$ in the Kaptein rule equation (Eq. 1). As shown in Fig. 1, the sign switch is observed in the case of HOPI (evidently for all the resonances) when the dye is altered from fluorescein to AT12. This finding can then be used to set the unknown $g$ factor of HOPI radicals between the $g$ factors of the dyes and in respect to the known values of tryptophan and tyrosine radicals as shown in Fig. 2. Moreover, the observation of the anomalous line signs in photo-CIDNP experiments monitored with TCBP in a previous study enabled us to rank the HOPI compounds with a $g$ factor between 2.0034 (fluorescein) and 2.0035 (TCBP) (Torres et al., 2021).

This is not only an elegant illustration of Kaptein's rules but also evidence of a $g$-factor evolution upon chemical modification from tryptophan to its oxidocyclization product HOPI (Fig. 1). Since the $g$ factor originates from spin-orbit coupling, the shape of the aromatic ring was suspected to be the main factor for an increased $g$ factor and improved polarizability. This hypothesis is consistent with the results previously obtained for tyrosine, which has a comparable aromatic system and is preferentially polarized in the presence of AT12 (Sobol et al., 2019). Therefore, the photo-CIDNP spectrum of 2,3-dihydro-tryptophan (dH-TRP), which has the same aromatic system as HOPI (Fig. 1), was recorded for both dyes, AT12 and fluorescein (Fig. 1). As expected, the polarization sign switch could be observed again upon dye change, confirming the idea that similar aromatic systems should or may yield close $g$ factors (as pinpointed to in Figs. 1 and 2).

However, the good photo-CIDNP performance of the HOPI compound is not observed for dH-TRP as the polarization enhancements in the presence of AT12 were only 10-fold and 17-fold in the presence of fluorescein (Table 1). This difference cannot be attributed to a slight difference in the $g$ factor (towards the $g$ factor of fluorescein), because the SNE in the presence of fluorescein did not compensate the loss in SNE in the presence of AT12 as would be expected if the enhancement would solely rely on a $g$-factor value change (Fig. 1 and Table 1).

\subsection{The involvement of side-chain properties in the photo-CIDNP performance of tryptophan and tyrosine derivatives}

The lower performances of dH-TRP in comparison to HOPI despite similar $g$ factors turned the focus to the potential involvement of the side chain. Prior work has been done by Saprygina et al. (2014), studying the influence of $\mathrm{N}$ acetylation on the quenching rate of TCBP. The replacement of the $\alpha$-amine by a $\mathrm{N}$-acetyl resulted in the vanishing of the positive charge and lower quenching rates of the triplet-state photosensitizer accompanied by lower time-resolved photoCIDNP enhancements and interpreted as causative of the Nacetylation. Similarly, to this approach, side-chain modifications of the same molecular species were studied in the context of CW-photo-CIDNP.

Here, first insights into the potential role of the side chain was gathered by a comparison of the tryptophan-derivative tryptamine (for chemical structure see Fig. 1) with tryptophan. Tryptamine differs from tryptophan by the absence of the carboxylic acid on its side chain. Indeed, improved CWphoto-CIDNP SNE is observed for tryptamine when compared with tryptophan, especially after irradiation in the presence of AT12 for which a further signal enhancement of a factor of 2 is documented (Fig. 1, Table 1). Since both molecules have the same aromatic system and thus similar magnetic parameters (Connor et al., 2008), and a similar reaction mechanism is expected (i.e., ET, electron transfer), the improved polarization of tryptamine might be due to the charge of the molecule that differs from tryptophan by the absence of the carboxylic acid on its side chain causing potentially a change in the quenching kinetics. Fluorescein contains a benzocarboxylate moiety of typical pKa 22.5 and a xanthenol of pKa 6.4, (Lavis et al., 2007) and is, in the buffer of interest, twice negatively charged. AT12 is neutral in the experimental conditions $(\mathrm{pH}=7.1)$; however, the aromatic system is globally carrying a positive charge (Fig. 1). Due to its overall positive charge, it is expected that the quenching of fluorescein by tryptamine is faster than by tryptophan, which is globally neutral in the experimental conditions. However, the strongest improvement in terms of CW-photo-CIDNP performances is for AT12-monitored experiments, despite the rather repulsive charges in play.

In order to elaborate further on the hypothesis of the direct potential impact of the charge of the side chain on the SNE of AT12-monitored CW-photo-CIDNP experiments, such experiments were conducted on tyramine (Table 1), which is a derivative of tyrosine where the $\alpha$-carboxylate moiety is absent. Tyrosine and tyramine are preferentially polarized upon irradiation in the presence of AT12 oppositely to tryptophan/tryptamine, due their higher $g$ factor, as shown in Fig. 2. The CW-photo-CIDNP SNE in the presence of AT12 is sig- 
TRP<smiles>[NH3+]C(Cc1c[nH]c2ccccc12)C(=O)[O-]</smiles>

TRPA<smiles>[NH3+]CCc1c[nH]c2cccc(I)c12</smiles>

IPA<smiles>O=C([O-])CCc1c[nH]c2ccccc12</smiles>

IAA<smiles>O=C([O-])Cc1c[nH]c2cccc(I)c12</smiles>

PEI<smiles>c1ccc2c(CCN3CC[NH2+]CC3)c[nH]c2c1</smiles>

HOPI<smiles>O=C([O-])C1CC2(O)c3cc(I)ccc3NC2[NH2+]1</smiles><smiles>[NH3+]C(CC1CNc2ccc(I)cc21)C(=O)[O-]</smiles>

TYR<smiles>[NH3+]C(Cc1ccc(O)c(I)c1)C(=O)[O-]</smiles>

TYRA<smiles>[Y5]CCc1cc(I)c(O)c(I)c1</smiles><smiles></smiles><smiles>CCOC(=O)c1ccccc1-c1c2ccc(=O)cc-2oc2cc(O)ccc12</smiles>

SNE

18

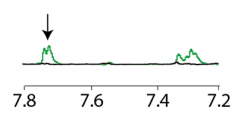

SNE

54

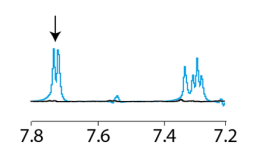

45

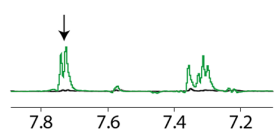

70

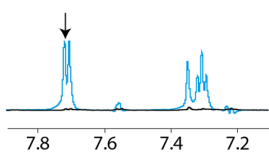

45

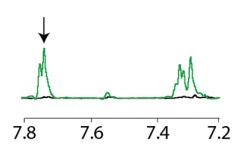

70

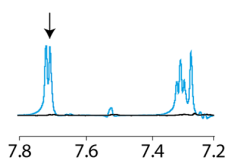

27

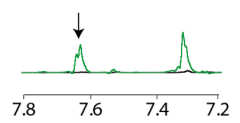

54

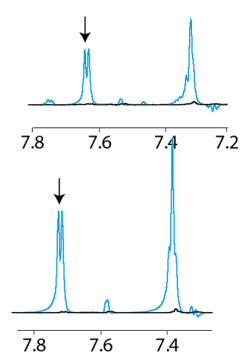

90

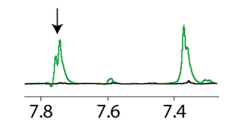

103

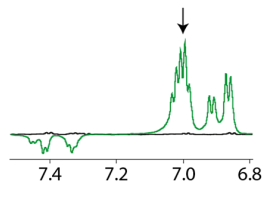

10

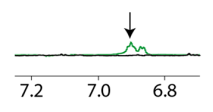

$-17$

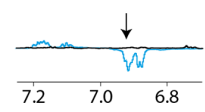

$-38$

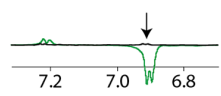

$-20$

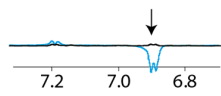

$-63$

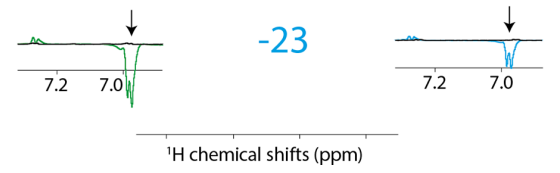

Figure 1. Photo-CIDNP spectra of tryptophan or tyrosine derivatives. The best aromatic proton's signal-to-noise enhancement (SNE) is provided, and the corresponding signal is pinpointed with an arrow. All spectra were recorded after $4 \mathrm{~s}$ of irradiation at $1 \mathrm{~W}$, in the presence of either AT12 $(20 \mu \mathrm{M}$, irradiation wavelength $532 \mathrm{~nm})$ or fluorescein $(20 \mu \mathrm{M}$, irradiation wavelength $450 \mathrm{~nm})$, the to-be-polarized molecule is present in a concentration of $100 \mu \mathrm{M}$. Color code: green are the AT12-monitored SNE values and photo-CIDNP spectra, blue are the fluorescein-monitored SNE values and photo-CIDNP spectra, and black are the non-irradiated reference spectra. The structures are provided in their ionization state at experimental conditions of $\mathrm{pH} 7.1$. 
Table 1. Signal enhancement of tryptophan and tyrosine derivatives $(100 \mu \mathrm{M})$ after irradiation in the presence of AT12 or fluorescein $(20 \mu \mathrm{M})$ at $1 \mathrm{~W}$ for $4 \mathrm{~s}$. The reported charges are of the diamagnetic molecules. The values for the tyrosine enhancement are taken from Sobol et al. (2019) and for the tryptophan and HOPI enhancement from Torres et al. (2021). Nevertheless, sample conditions were identical. The $\log (P)$ is the logarithm of the partition coefficient $P$, and $P$ is the ratio of the concentration of the compounds in a mixture of the two immiscible solvents octanol and water $\left(P=[\text { molecule }]_{\text {octanol }} /[\text { molecule }]_{\text {water }}\right)$.

\begin{tabular}{lrrrr}
\hline Molecule & AT12 SNE & Fluorescein SNE & Charge & $\log (P)$ \\
\hline TRP & $18^{\mathrm{a}}$ & $54^{\mathrm{a}}$ & 0 & -1.1 \\
TRPA & 45 & 70 & +1 & 1.6 \\
TYR & $-38^{\mathrm{b}}$ & $-20^{\mathrm{b}}$ & 0 & -2.3 \\
TYRA & -63 & -23 & +1 & 1.1 \\
HOPI & $90^{\mathrm{a}}$ & $-70^{\mathrm{a}}$ & 0 & -2.1 \\
dH-TRP & 10 & -17 & 0 & -1.5 \\
PEI & 44 & 103 & +1 & 1.9 \\
IPA & 45 & 70 & -1 & 1.8 \\
IAA & 27 & 54 & -1 & 1.4 \\
\hline
\end{tabular}

a Torres et al. (2021); ${ }^{\mathrm{b}}$ Sobol et al. (2019).

nificantly higher for tyramine when compared with tyrosine (Fig. 1, Table 1). The minor SNE enhancement for tyramine versus tyrosine photo-CIDNP experiments monitored by fluorescein could be explained by the different $\Delta g$. This experiment supports the finding that the chemical modification of side chains can significantly improve the SNE for CWphoto-CIDNP in the presence of AT12. Next, the CW-photoCIDNP spectra of indole propionic acid (IPA) and indole acetic acid (IAA) have been recorded. IPA is the negatively charged analogue of tryptophan (Table 1) where the $\alpha$-amine is lacking. IAA is similar to IPA but the carboxylate group is closer to the aromatic ring, since it is in the $\beta$ position. Unexpectedly, the IPA yielded the same SNE as tryptamine (Table 1), whereas an interpretation of the SNE solely based on the charge, overall negative for IPA, predicted an opposite effect on the performance as compared to the positively charged tryptamine. Despite identical charge as IPA, IAA (Table 1) exhibits comparable performances as compared to tryptophan. Moreover, 3-(2-(piperazin)ethyl)-indole (PEI) is an analogue of tryptamine where the $\alpha$-amine is replaced by a piperazin moiety. In PEI, the overall charge is similar to tryptamine since the pKa of the tertiary amine (of the ground state) is close to 4 and only the secondary amine is positively charged, due to its pKa around 9 . PEI yielded similar polarization performances as tryptamine upon irradiation in the presence of AT12 and showed even higher SNE for fluorescein-monitored photo-CIDNP experiments. An interpretation of these results solely based on the respective overall charges therefore fails to draw any trend.

Alternatively, it could be hypothesized that a different side-chain dynamic may play a role in the SNE of CWphoto-CIDNP. With the side-chain alterations, not only the charge of the side chain changed but also the dynamics with tryptamine, IPA, IAA, PEI, and tyramine comprise faster side-chain motion than tryptophan and tyrosine. This change in dynamics is indicated by the observation that the $\mathrm{H}_{\beta}$ res- onances are split for tryptophan and not for tryptamine, IPA, IAA or PEI (Fig. 3). The same degeneracy of the $\mathrm{H}_{\beta}$ chemical shifts is observed when the amine group is removed from the tyrosine to become the tyramine (Fig. 3). However, this hypothesis is not supported experimentally since the dihydro-tryptophan, which has a higher side-chain mobility as compared to the HOPI, shows less polarization than HOPI.

The only summary of this first attempt to interpret the chemical space exploration is that the simultaneous presence of the $\alpha$-carboxylate and the $\alpha$-amine is suboptimal for CW-photo-CIDNP SNE when monitored with fluorescein or AT12 as supported by the less polarization properties of tryptophan, tyrosine and dH-TRP when compared with their analogues. A corollary of the presence of these $\alpha$-carboxylate and $\alpha$-amine compounds is the water solubility of the small molecules and their solvation shells. This idea brings us to another difference between the different side-chain properties, which is hydrophobicity. This can be assessed with the logarithm of the calculated partition coefficient between octanol and water, $\log (P)$. The evolution of the hydrophobicity within the different families of compounds and its influence on the CW-photo-CIDNP performances was therefore investigated. Within the tryptophan derivative group, i.e., tryptophan, dihydro-tryptophan, tryptamine, IAA, IPA and PEI, the increasing hydrophobicity is beneficial to the CW-photoCIDNP performances when monitored by both fluorescein and AT12 dyes (Fig. 4a and b). The same trend is suggested for the tyrosine derivatives tyrosine and tyramine: tyramine, which is more hydrophobic, is better polarized, especially in the presence of AT12, than tyrosine (Table 1). HOPI was not included in this analysis since it is rather far away from the chemical space of the two series of interest.

The positive influence of hydrophobicity on the SNE may be explained by two distinct mechanisms that also may work in concert. First, the aromatic nature of the dye-molecule in- 


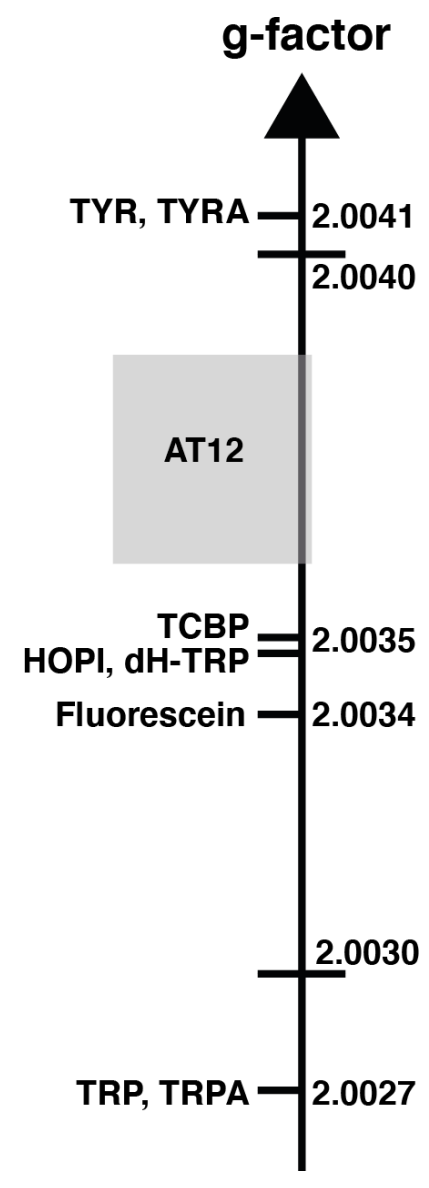

Figure 2. The sign alternation enables us to locate the molecules on the $g$-value scale. The ranking of HOPI and dH-TRP provides fine-scale information about their radical $g$ values. For the abbreviations that are not defined elsewhere, TRP stands for tryptophan, TRPA stands for tryptamine, TYR is tyrosine and TYRA is tyramine. (Figure was adapted from Sobol et al., 2019.)

teraction is favored for more hydrophobic molecules. Second, the water shell surrounding the molecule is perturbed by the different hydrophobicity conditions of the side chains as can be observed from the $\mathrm{H}_{\beta}$ dynamics (Fig. 3). Hence, the $\pi-\pi$ stacking between the excited dye and the molecule, and therefore the orbital overlap, could be altered in a positive manner by increasing the hydrophobicity of the molecule. In summary, the hydrophobicity variation upon side-chain modification appears to have a qualitative impact on the CWphoto-CIDNP SNE unlike the charge and dynamic variation. In other words, the observed trends suggest a positive impact on the SNE for higher hydrophobicity of the molecules sharing a common aromatic moiety. While noting these findings, it must be stated (as above) that the exact nature of the polarization can only be determined by TR-photo-CIDNP. However, the presented empirical approach is regarded informative for CW-photo-CIDNP applications as the positive aspects of CW-photo-CIDNP with several seconds of light irradiation in terms of signal to noise and easy and cheap setup is apparent. The importance of the irradiation time is indicated in the Supplement Fig. 1, yielding for both compounds tested (i.e., HOPI and TRP) an enhanced signal by a factor of 1.5 to more than 2 between 1 and $4 \mathrm{~s}$ irradiation time.

In addition, the $\mathrm{pH}$ dependencies of the photo-CIDNP SNE were recorded for the different tryptophan analogues, in a $\mathrm{pH}$ range between 5 and 9 within $2 \mathrm{pH}$ units of physiological conditions. While the pKa of the indole is typically around 16, the pKa of the indolyl group has been observed to be rather in the range 7-8 (Stob and Kaptein, 1989; Hore and Broadwurst, 1993). The latter pKa is known to have a significant influence on the photo-CIDNP hyperpolarization performances. The $\mathrm{pH}$-dependent photo-CIDNP performances of the five tryptophan analogues (TRP, TRPA, PEI, IPA and IAA) for both dyes show overall similar behaviors but group, in the presence of fluorescence, into TRP and TRPA with a maximum already at $\mathrm{pH} 7$ and IPA, PEI and IAA with a maximum at $\mathrm{pH} 8$, while the relative enhancement of IAA at $\mathrm{pH} 7$ is significantly lower when compared with the other compounds (Fig. S2b). In the presence of AT12, the differences are less obvious with a maximum enhancement reached for pH $>9$ (Fig. S2b). Similar results have been observed by Stob and Kaptein, for tryptophan and $\mathrm{N}$-acetyl tryptophan. Importantly, the $\log (P)$ dependencies were still observed at the optimal $\mathrm{pH}$ abovementioned as the plots in Fig. S3 show similar correlations as observed in Fig. 4, with the difference that the IPA and IAA show the best enhancements for the photo-CIDNP spectra monitored with AT12 and fluorescein. While the common features between these two compounds is the carboxylic acid ending the side chain, the ionic interaction with the dye cannot simply explain these better performances, as the dye charges are significantly different (Fig. 1). Moreover, the significantly better SNE for PEI as compared to TRPA and TRP is in favor of a positive influence of the hydrophobicity. The extension of this hypothesis to a broader set of molecules may be beneficial to confirm the positive impact of the carboxylic acid in comparison to other negatively charged molecules and the impact of hydrophobicity on the photo-CIDNP performances.

\section{Conclusion}

Photo-CIDNP appears to be an interesting approach for NMR signal enhancement through polarization with the potential to rescue the low sensitivity usually inherent with NMR. The small library of CW-photo-CIDNP-active compounds presented indicates the existence of many photoCIDNP-active molecules in the known chemical space, mainly described in two prior studies (Stob and Kaptein, 1989; Hore and Broadwurst, 1993). The initial qualitative physicochemical analysis of the 10 compounds studied indicates that side chains of the different molecules play a key 


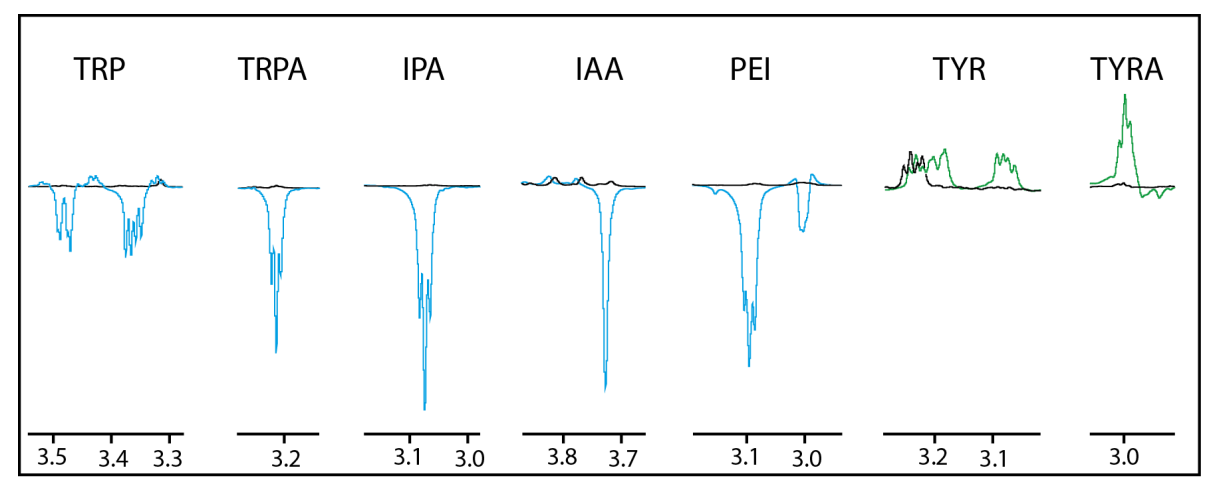

${ }^{1} \mathrm{H}$ chemical shifts $(\mathrm{ppm})$

Figure 3. $\mathrm{H}_{\beta}$ anomalous signals for the different molecules. Black lines are the reference spectra, and blue lines are the irradiated spectra in the presence of fluorescein, and the green lines are the irradiated spectra in the presence of AT12. Scales between the different molecules are not respected for clarity purposes.
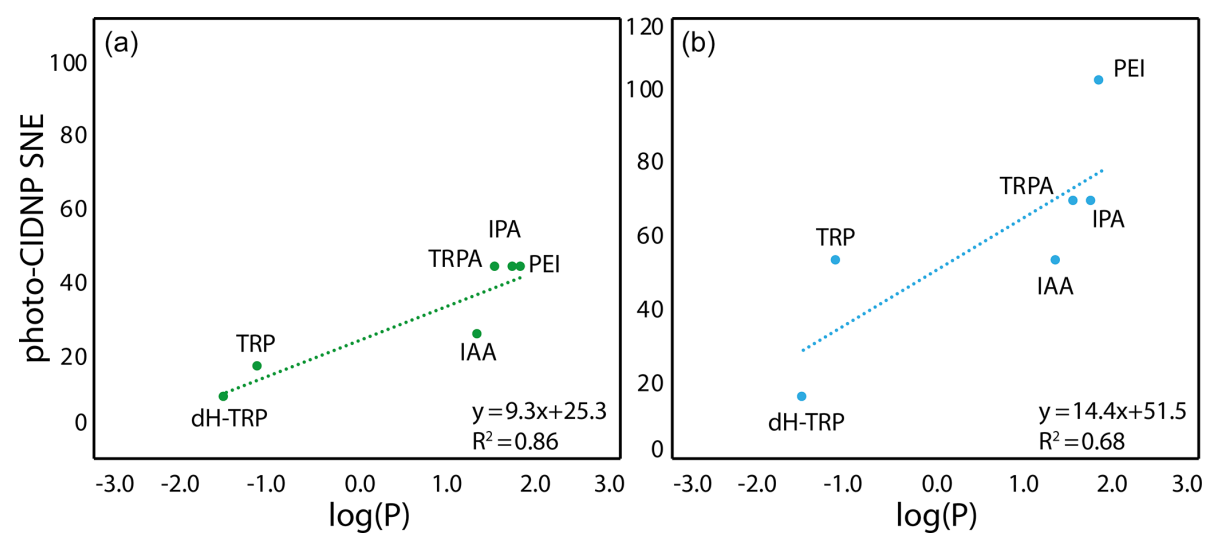

Figure 4. Correlation between the molecule hydrophobicity and the photo-CIDNP performances for the tryptophan derivatives. (a) In the case where CW-photo-CIDNP is monitored by AT12. (b) In the case where CW-photo-CIDNP is monitored by fluorescein. The $\log (P)$ is the logarithm of the partition coefficient, $P$, between octanol and water, and values were calculated with DataWarrior ${ }^{\circledR}$. A statistical analysis of the trend lines using Pearson's $R$ coefficient and Student's $t$ test for the hypothesis shows that for AT12 (a) a $t$ of 4.87 and a $p$ value of 0.008 are obtained, and for fluorescein (b) a $t$ of 2.6 and a $p$ value of 0.060 are obtained. Hence, in both cases, the hypothesis H0 (absence of correlation) is rejected, and the alternative hypothesis (correlation is non-null) is retained.

role in the CW-photo-CIDNP SNE, which could not be predicted by solely considering the ionic interaction monitoring the dye quenching or the radical pair stabilization. Rather, the hydrophobicity of the molecules revealed an influence on SNE, because the polarization performances improved gradually within the two classes of derivatives with hydrophobicity. Importantly, the pKa of the indolyl radical is a critical parameter for the optimization of the polarization effect of photo-CIDNP. For indole groups, the optimal $\mathrm{pH}$ is above 8.0 for fluorescein and 9.0 for AT12; the measurements at higher $\mathrm{pH}$ improve in particular the polarization of the analogues carrying a carboxylic acid, which were found to be performing best in basic conditions. The importance of the hydrophobicity as one signature opens the way for simple chemical space exploration, since it can be simply assessed by its $\log (P)$. This allows for the possibility of empiri- cally screening the chemical space for potential highly active CW-photo-CIDNP small molecules. From this initial study emerges the potential of making CW-photo-CIDNP a much broader method for signal enhancement in the biomedical NMR field and the request for the exploration of the nonaromatic chemical space within photo-CIDNP-active molecules.

\section{Material and methods}

The NMR measurements were performed at $298 \mathrm{~K}$ on a Bruker Avance III $600 \mathrm{MHz}$ spectrometer equipped with a cryoprobe. The irradiation of AT12 samples was performed with a Coherent Verdi V10 diode-pumped solid-state laser emitting at a wavelength of $532 \mathrm{~nm}$. The laser used for the fluorescein samples was a Thorlabs L450P1600MM, which is a diode laser emitting at $450 \mathrm{~nm}$. The laser light was cou- 
pled (using appropriate coupling optics) into an optical fiber (Thorlabs, FG950UEC) of length $10 \mathrm{~m}$ and a diameter of $0.95 \mathrm{~mm}$. The end of the fiber was inserted into the sample solution in a $3 \mathrm{~mm}$ NMR tube to a depth of about $5 \mathrm{~mm}$ above the NMR coil region.

Tyrosine, tyramine, tryptophan, tryptamine, IPA and IAA were purchased from Sigma; dH-TRP was purchased from Akos Pharma; and PEI was purchased from ChemSpace LLC. HOPI was synthesized in-house according to the previously published protocol (Torres et al., 2021). Glucose oxidase (GO) and catalase (Cat) were prepared as stock solutions of 0.2 and $0.18 \mathrm{mg} / \mathrm{mL}$, respectively, in a $0.1 \mathrm{M}$ sodium/potassium phosphate buffer $(\mathrm{pH} 7.1)$ with $5 \% \mathrm{D}_{2} \mathrm{O}$. The stock solution of Atto Thio 12 (AT12) was $1 \mathrm{mg} / \mathrm{mL}$ in $\mathrm{H}_{2} \mathrm{O}$. To prevent dye quenching, the enzyme cocktail glucose oxidase (GO, $120 \mathrm{kDa})$, catalase (Cat, $240 \mathrm{kDa}$ ) and Dglucose $(\mathrm{G}, 180 \mathrm{Da})$ was used at a concentration of $14 \mathrm{nM}$ for each enzyme and $2.5 \mathrm{mM}$ of glucose, as described elsewhere (Okuno and Cavagnero, 2016; Lee and Cavagnero, 2013). The stock solutions were $0.25 \mu \mathrm{M}$ for GO and $0.16 \mu \mathrm{M}$ for Cat, respectively. The glucose stock solution was $500 \mathrm{mM}$ in $\mathrm{D}_{2} \mathrm{O}$ with $0.02 \% \mathrm{NaN}_{3}$. All the samples were prepared in a $100 \mathrm{mM} \mathrm{KPO}_{4}$ buffer at $\mathrm{pH}=7.1$ with either $20 \mu \mathrm{M}$ AT 12 or $25 \mu \mathrm{M}$ fluorescein and $100 \mu \mathrm{M}$ target molecule. The $\mathrm{pH}$ titrations where performed in the same buffer, with the adjusted $\mathrm{pH}(5.0,6.0,7.0,8.0,9.0)$, and the oxygen scavenging was performed using a cycle of vacuum and nitrogen atmosphere flush, for $30 \mathrm{~min}$.

Data availability. The data related to the present work are available upon request.

Supplement. The supplement related to this article is available online at: https://doi.org/10.5194/mr-2-321-2021-supplement.

Author contributions. FT performed the experiments, data analysis and wrote the article. AR helped with experiments, data analysis and writing. RR performed data analysis and wrote the article.

Competing interests. The authors declare that they have no conflict of interest.

Special issue statement. This article is part of the special issue "Robert Kaptein Festschrift". It is not associated with a conference.

Acknowledgements. We would like to acknowledge ETH for financial support.
Review statement. This paper was edited by Jörg Matysik and reviewed by Peter Hore and three anonymous referees.

\section{References}

Ardenkjaer-Larsen, J. H., Boebinger, G. S., Comment, A., Duckett, S., Edison, A. S., Engelke, F., Griesinger, C., Griffin, R. G., Hilty, C., Maeda, H., Parigi, G., Prisner, T., Ravera, E., van Bentum, J., Vega, S., Webb, A., Luchinat, C., Schwalbe, H., and Frydman, L.: Facing and Overcoming Sensitivity Challenges in Biomolecular NMR Spectroscopy, Angew. Chem. Int. Edit., 54, 9162-9185, https://doi.org/10.1002/anie.201410653, 2015.

Bargon, J., Fischer, H., and Johnsen, U.: KernresonanzEmissionslinien Wahrend Rascher Radikalreaktionen .I. Aufnahmeverfahren Und Beispiele, Z. Naturforsch. Pt. A, 22, 1551-1555, 1967.

Bernarding, J., Euchner, F., Bruns, C., Ringleb, R., Mueller, D., Trantzschel, T., Bargon, J., Bommerich, U., and Plaumann, M.: Low-cost LED-based Photo-CIDNP Enables Biocompatible Hyperpolarization of ${ }^{19} \mathrm{~F}$ for $\mathrm{NMR}$ and MRI at $7 \mathrm{~T}$ and $4.7 \mathrm{~T}$, Chemphyschem, 19, 2453-2456, https://doi.org/10.1002/cphc.201800570, 2018.

Closs, G. L.: Mechanism explaining nuclear spin polarizations in radical combination reactions, J. Am. Chem. Soc., 91, 45524554, 1969.

Closs, G. L. and Trifunac, A. D.: Chemically Induced Nuclear Spin Polarization as a Tool for Determination of Spin Multiplicities of Radical-Pair Precursors, J. Am. Chem. Soc., 91, 4554-4555, https://doi.org/10.1021/Ja01044a044, 1969.

Connor, H. D., Sturgeon, B. E., Mottley, C., Sipe, H. J., and Mason, R. P.: L-tryptophan radical cation electron spin resonance studies: Connecting solution-derived hyperfine coupling constants with protein spectral interpretations, J. Am. Chem. Soc., 130, 6381-6387, https://doi.org/10.1021/ja0780277, 2008.

Goez, M.: An introduction to chemically induced dynamic nuclear polarization, Concepts in Mag. Reson. Part A, 7, 69-86, 1995.

Hore, P. J. and Broadhurst, R. W.: Photo-Cidnp of Biopolymers, Prog. Nucl. Mag. Res. Sp., 25, 345-402, 1993.

Hore, P. J., Zuiderweg, E. R. P., Kaptein, R., and Dijkstra, K.: Flash-Photolysis NMR - Cidnp Time-Dependence in Cyclic Photochemical-Reactions, Chem. Phys. Lett., 83, 376-383, https://doi.org/10.1016/0009-2614(81)85483-8, 1981.

Hore, P. J., Winder, S. L., Roberts, C. H., and Dobson, C. M.: Stopped-Flow Photo-CIDNP Observation of Protein Folding, J. Am. Chem. Soc., 119, 5049-5050, 1997.

Kaptein, R.: Simple rules for chemically induced dynamic nuclear polarization, J. Chem. Com. D: Chem. Com., 732-733, https://doi.org/10.1039/c29710000732, 1971.

Kaptein, R. and Oosterhoff, J. L.: Chemically induced dynamic nuclear polarization II: (Relation with anomalous ESR spectra), Chem. Phys. Lett., 4, 195-197, https://doi.org/10.1016/00092614(69)80098-9, 1969.

Kaptein, R., Dijkstra, K., and Nicolay, K.: Laser Photo-Cidnp as a Surface Probe for Proteins in Solution, Nature, 274, 293-294, https://doi.org/10.1038/274293a0, 1978.

Kuhn, L.: Hyperpolarization methods in NMR spectroscopy, Top. Curr. Chem., 338, Springer Berlin Heidelberg, 229-300, https://doi.org/10.1007/128_2013_427, 2013. 
Kuprov, I. and Hore, P. J.: Chemically amplified ${ }^{19} \mathrm{~F}-{ }^{1} \mathrm{H}$ nuclear Overhauser effects, J. Magn. Reson., 168, 1-7, https://doi.org/10.1016/j.jmr.2004.01.011, 2004.

Kuprov, I., Craggs, T. D., Jackson, S. E., and Hore, P. J.: Spin relaxation effects in photochemically induced dynamic nuclear polarization spectroscopy of nuclei with strongly anisotropic hyperfine couplings, J. Am. Chem. Soc., 129, 9004-9013, https://doi.org/10.1021/ja0705792, 2007.

Lavis, L. D., Rutkoski, T. J., and Raines, R. T.: Tuning the $\mathrm{p} K_{\mathrm{a}}$ of fluorescein to optimize binding assays, Anal. Chem., 79, 67756782, https://doi.org/10.1021/ac070907g, 2007.

Lee, J. H. and Cavagnero, S.: A Novel Tri-Enzyme System in Combination with Laser-Driven NMR Enables Efficient Nuclear Polarization of Biomolecules in Solution, J. Phys. Chem. B, 117, 6069-6081, https://doi.org/10.1021/jp4010168, 2013.

Mok, K. H. and Hore, P. J.: Photo-CIDNP NMR methods for studying protein folding, Methods, 34, 75-87, https://doi.org/10.1016/j.ymeth.2004.03.006, 2004.

Mok, K. H., Nagashima, T., Day, I. J., Jones, J. A., Jones, C. J., Dobson, C. M., and Hore, P. J.: Rapid sample-mixing technique for transient NMR and photo-CIDNP spectroscopy: applications to real-time protein folding, J. Am. Chem. Soc., 125, 12484-12492, https://doi.org/10.1021/ja036357v, 2003.

Morozova, O. B. and Ivanov, K. L.: Time-Resolved Chemically Induced Dynamic Nuclear Polarization of Biologically Important Molecules, Chemphyschem, 20, 197-215, https://doi.org/10.1002/cphc.201800566, 2019.

Morozova, O. B., Yurkovskaya, A. V., Vieth, H. M., and Sagdeev, R. Z.: Intramolecular electron transfer in tryptophan-tyrosine peptide in photoinduced reaction in aqueous solution, J. Phys. Chem. B, 107, 1088-1096, https://doi.org/10.1021/jp0265283, 2003.

Morozova, O. B., Hore, P. J., Sagdeev, R. Z., and Yurkovskaya, A. V.: Intramolecular electron transfer in lysozyme studied by time-resolved chemically induced dynamic nuclear polarization, J. Phys. Chem. B, 109, 21971-21978, https://doi.org/10.1021/jp053394v, 2005.

Morozova, O. B., Kiryutin, A. S., Sagdeev, R. Z., and Yurkovskaya, A. V.: Electron transfer between guanosine radical and amino acids in aqueous solution. 1. Reduction of guanosine radical by tyrosine, J. Phys. Chem. B, 111, 7439-7448, https://doi.org/10.1021/jp067722i, 2007.

Morozova, O. B., Kiryutin, A. S., and Yurkovskaya, A. V.: Electron transfer between guanosine radicals and amino acids in aqueous solution. II. Reduction of guanosine radicals by tryptophan, J. Phys. Chem. B, 112, 2747-2754, https://doi.org/10.1021/jp0752318, 2008.

Morozova, O. B., Ivanov, K. L., Kiryutin, A. S., Sagdeev, R. Z., Kochling, T., Vieth, H. M., and Yurkovskaya, A. V.: Timeresolved CIDNP: an NMR way to determine the EPR parameters of elusive radicals, Phys. Chem. Chem. Phys., 13, 6619-6627, https://doi.org/10.1039/c0cp02449j, 2011.
Morozova, O. B., Panov, M. S., Fishman, N. N., and Yurkovskaya, A. V.: Electron transfer vs. proton-coupled electron transfer as the mechanism of reaction between amino acids and triplet-excited benzophenones revealed by time-resolved CIDNP, Phys. Chem. Chem. Phys., 20, 21127-21135, https://doi.org/10.1039/c8cp03591a, 2018.

Okuno, Y. and Cavagnero, S.: Fluorescein: A Photo-CIDNP Sensitizer Enabling Hypersensitive NMR Data Collection in Liquids at Low Micromolar Concentration, J. Phys. Chem. B, 120, 715723, https://doi.org/10.1021/acs.jpcb.5b12339, 2016.

Okuno, Y. and Cavagnero, S.: Photochemically Induced Dynamic Nuclear Polarization: Basic Principles and Applications, Emagres, 6, 283-313, https://doi.org/10.1002/9780470034590.emrstm1499, 2017.

Saprygina, N. N., Morozova, O. B., Grampp, G., and Yurkovskaya, A. V.: Effect of amino group charge on the photooxidation kinetics of aromatic amino acids, J. Phys. Chem. A, 118, 339-349, https://doi.org/10.1021/jp4097919, 2014.

Sobol, A., Torres, F., Aicher, A., Renn, A., and Riek, R.: Atto Thio 12 as a promising dye for photo-CIDNP, J. Chem. Phys., 151, 234201, https://doi.org/10.1063/1.5128575, 2019.

Stob, S. and Kaptein, R.: Photo-Cidnp of the Amino-Acids, Photochem. Photobiol., 49, 565-577, https://doi.org/10.1111/j.17511097.1989.tb08425.x, 1989.

Torres, F., Sobol, A., Greenwald, J., Renn, A., and Riek, R.: Molecular features toward high photo-CIDNP hyperpolarization explored through the oxidocyclization of tryptophan, Phys. Chem. Chem. Phys., 23, 6641-6650 https://doi.org/10.1039/D0CP06068B, 2021.

Tsentalovich, Y. P., Morozova, O. B., Yurkovskaya, A. V., Hore, P. J., and Sagdeev, R. Z.: Time-resolved CIDNP and laser flash photolysis study of the photoreactions of $N$-acetyl histidine with 2,2'-dipyridyl in aqueous solution, J. Phys. Chem. A, 104, 69126916, https://doi.org/10.1021/jp000019o, 2000.

Tsentalovich, Y. P., Lopez, J. J., Hore, P. J., and Sagdeev, R. Z.: Mechanisms of reactions of flavin mononucleotide triplet with aromatic amino acids, Spectrochim. Acta A, 58, 2043-2050, https://doi.org/10.1016/S1386-1425(01)00652-7, 2002.

Ward, H. R. and Lawler, R. G.: Nuclear Magnetic Resonance Emission and Enhanced Absorption in Rapid Organometallic Reactions, J. Am. Chem. Soc., 89, 55185519, https://doi.org/10.1021/Ja00997a078, 1967. 